\title{
Editorial
}

\section{Recent Trends of Hormone Receptor Research and New Strategy of Endocrine Therapy for Breast Cancer}

Breast Cancer 14:174, 2007.

Key words: Hormone receptor, Endocrine therapy, Estrogen receptor
Masafumi Kurosumi

Department of Pathology, Saitama Cancer Center, Japan.
Recently endocrine therapy of breast cancer has been changed because of the introduction of aromatase inhibitors for postmenopausal patients. In addition, examination methods of hormone receptor status have become unified in the immunohistochemical method and optimal evaluation criteria have been found necessary. On the other hand, fundamental research as to function of stromal cells of breast tissue has focused on the relation to postmenopausal estrogen secretion. In this special issue, we focus on recent trends of fundamental research about estrogen production in the breast tissue, methods of immunohistochemical evaluation of hormone receptor status and recent strategy of endocrine therapies for breast cancer patients.

In the first review, Dr. Yamaguchi explained about the microenvironmental regulation of estrogen function in breast cancer tissue and demonstrated the relationship of activity of ER and mechanism of cancer proliferation. In addition, the mechanism of aromatase production by stromal fibroblast and growth of breast cancers was precisely explained. In the second review, Dr. Oyama described the effects of fixation and processing for adequate immunohistochemical detection of hormone receptors in breast cancer and recommended performing suitable handling of tissues and staining for accurate evaluation of hormone receptor status in routine practice. In the third review, I described two scoring systems for immunohistochemical assessment of hormone receptor status. The utility of the J-Score based on the cutoff value of St. Gallen consensus meeting was presented and explained. In the fourth review, Dr.
Utsumi reviewed the recent perspectives of endocrine therapy for breast cancer and demonstrated the recent strategy of endocrine therapy based on the menopausal status and stage of disease. In the fifth review, Dr. Kurebayashi described the current clinical trials on endocrine therapy for breast cancer and individually explained the fields of chemoprevention, preoperative treatment, postoperative adjuvant treatment and treatment for recurrent diseases.

This special project is particularly important to understand recent information concerning the relationship between the function of hormone receptors and the effectiveness of endocrine therapy.

Reprint requests to Masafumi Kurosumi, Department of Pathology, Saitama Cancer Center, 818 Komuro, Ina-machi, Kitaadachi-gun, Saitama, 362-0806, Japan

E-mail: mkurosumi@cancer-c.pref.saitama.jp 